University of Wollongong

Research Online

Faculty of Engineering - Papers (Archive)

Faculty of Engineering and Information

Sciences

$1-1-2009$

\title{
Mechanism for initiating secondary currents in channel flows
}

Shuqing Yang

University of Wollongong, shuqing@uow.edu.au

Follow this and additional works at: https://ro.uow.edu.au/engpapers

Part of the Engineering Commons

https://ro.uow.edu.au/engpapers/3676

\section{Recommended Citation}

Yang, Shuqing: Mechanism for initiating secondary currents in channel flows 2009, 1506-1516.

https://ro.uow.edu.au/engpapers/3676

Research Online is the open access institutional repository for the University of Wollongong. For further information contact the UOW Library: research-pubs@uow.edu.au 


\title{
Mechanism for initiating secondary currents in channel flows
}

\author{
Shu-Qing Yang
}

\begin{abstract}
This study investigates the underlying mechanisms that initiate secondary flow in developing turbulent flow along a corner. This is done by theoretical examination of the total shear stress, which is the time-averaged product of instantaneous streamwise velocity $U$ and the velocity $V_{\mathrm{n}}$ normal to the interface. The study shows that lines of zero total shear stress exist in the flow region, which delineate the region of secondary flow. Therefore, the flow region is dividable and eight vortices occur in a duct flow. The theoretical and experimental results show that the division line, separating the neighboring secondary currents in a corner, is not always identical to the bisector of the corner, but deviates from the corner bisector if the aspect ratio is $b / h \neq 1$. By simplifying Reynolds equation in the near-bed region, we find that theoretically a lateral variation of streamwise velocity initiates the wall-tangent flow that drives the vortex in the region bounded by zero total shear stress. A simplified method for estimating the vortex center, near-bed secondary velocity, and shape of secondary currents has been proposed, and a good agreement between the measured and predicted features is achieved.
\end{abstract}

Key words: dip-phenomenon, division line, Reynolds shear stress, secondary currents of Prandtl's second kind, turbulent energy, velocity distribution.

Résumé : La présente étude examine les mécanismes sous-jacents qui initient un écoulement secondaire dans un écoulement turbulent en développement le long d'un coin. Cela est accompli en examinant de manière théorique la contrainte totale en cisaillement, laquelle est le produit pondéré dans le temps de la composante longitudinale instantanée de la vitesse $U$ et la vitesse $V_{n}$ perpendiculaire à l'interface. L'étude montre que, dans la région de l'écoulement, il existe des lignes de zéro contrainte totale de cisaillement qui délimitent la région d'écoulement secondaire. C'est pourquoi la région d'écoulement peut être divisée et que huit vortex surviennent dans un écoulement en canalisation. Les résultats théoriques et expérimentaux montrent que la ligne de division, séparant les courants secondaires avoisinants dans un coin, n'est pas toujours identique à la bissectrice du coin, mais qu'elle dévie de la bissectrice du coin si le rapport de forme $b / h \neq 1$. En simplifiant l'équation de Reynolds dans la région près du lit, les résultats théoriques montrent qu'une variation latérale de la composante longitudinale de la vitesse initie l'écoulement tangent au mur qui génère le vortex dans la région limitée par la contrainte totale de cisaillement zéro. Une méthode simplifiée pour estimer le centre du vortex, la vitesse secondaire près du lit et la forme des courants secondaires a été proposée et une bonne corrélation a été obtenue entre les paramètres mesurés et prédits.

Mots-clés : phénomène de plongée, ligne de division, contrainte de cisaillement Reynolds, courants secondaires de second type de Prandtl, énergie turbulente, distribution de la vitesse.

[Traduit par la Rédaction]

\section{Introduction}

The motion of fluids in ducts of rectangular cross section is of theoretical and practical interest, and its mechanism driving secondary currents has motivated many researchers. Nikuradse (1926) first made the observations of secondary currents that appeared near the corners of the duct cross section, leading to a deformation of the mean velocity contours. Such corner vortices in a straight noncircular duct flow have been classified as secondary flows of Prandtl's second kind, whereas the secondary currents of the first kind arise from the

Received 18 May 2008. Revision accepted 19 May 2009.

Published on the NRC Research Press Web site at cjce.nrc.ca on 6 October 2009.

S. Yang. School of Civil Mining \& Environmental Engineering, University of Wollongong, NSW 2522, Australia (email: shuqing@uow.edu.au).

Written discussion of this article is welcomed and will be received by the Editor until 31 January 2010. skewing of the mean flow in curved channels, for which secondary velocities are usually one order of magnitude higher. For the secondary currents of the second kind caused by the sidewall effect, even though secondary velocities usually amount to a few percent of the bulk velocity, the presence of secondary flow displaces considerably the velocity contours. Thus, for any attempt to deal with duct flow, one must pay special attention to the effect of this type of secondary flows.

Previous works are separated into two groups. The first is the experimental investigations following Nikuradse's observations: a large number of experimental studies have followed Nikuradse by means of velocity measurements or flow visualization techniques, such as Brundrett and Baines (1964), Tracy (1965), Gessner (1973), Melling and Whitelaw (1976), Perkins (1970), Sata et al. (1994), and Faisst and Eckhardt (2003). The second studies are the theoretical approaches, which were started by Einstein and Li (1958) who deduced the equation of relation between the longitudinal vorticity and Reynolds shear stress. It is accepted widely that secondary flows are induced by the imbalance of nor- 
mal Reynolds shear stress, i.e., $\left(\overline{v^{\prime 2}}-\overline{w^{\prime 2}}\right)$ in the cross-sectional plane (Hinze 1973; Gerard 1978). Speziale (1982) has introduced a sufficient condition for the development of longitudinal vortices stating that the longitudinal velocity field must give rise to a difference in the normal stresses for the secondary flow to appear. As previous researchers attributed the generation of secondary currents in a duct flow to the normal stresses, rather than the mean longitudinal velocity that can be readily determined, the evaluation of normal stresses, $\left(\overline{v^{\prime 2}}-\overline{w^{\prime 2}}\right)$ needs to be measured experimentally or computed numerically using sophisticated equipments or models. The first calculation of secondary currents in straight noncircular ducts was carried out by Launder and Ying (1972) who used the algebraic stress model. Several refined turbulence models, based on theirs, have been proposed, namely the length-scale model (Gessner and Emery 1981), large-eddy simulations (e.g., Madabhushi and Vanka 1991), and direct numerical simulations (Gavrilakis 1992). These numerical studies show that inside a duct flow, there always exist eight vortices, symmetric about the bisectors of each corner. However, it is worthwhile to point out that it is not always true that two neighboring secondary currents are separated by the bisector of the base angle. Tracy's (1965) measurement in a rectangular duct shows that the turbulent characteristics are different on both sides of $y=1.2 z$, rather than the bisector, i.e., $y=z$ (Fig. 1).

Contrary to the foregoing explanation, Gessner (1973) discovered that, after experimentally examining the normal stress balance along a corner bisector, the anisotropy of the turbulent normal stresses, $\overline{v^{\prime 2}}$ and $\overline{w^{\prime 2}}$, did not play a major role in the generation of secondary currents because all the terms in the equation derived by Einstein and Li (1958) are at least an order of magnitude smaller than the terms involving vorticity. He inferred from his experimental data that the secondary motion is initiated and directed toward the corner as a direct result of turbulent shear stress gradients in the main flow region, not the imbalance of normal stresses. Gessner's experimental conclusion was surprising since it totally played down the role of the anisotropy of the turbulent normal stresses. Thereafter, many experimental results support Gessner's conclusion, for example, Galletti and Bottaro (2004), Bottaro et al. (2006), etc.

Numerical modelers (Gavrilakis 1992; Mompean et al. 1996; Mompean 1998) also found that the previous numerical solutions of turbulence closure models have underestimated consistently the magnitudes of the secondary flows when compared with the results produced by direct simulation. Speziale (1987) claimed that “...models of turbulence can give rise to highly inaccurate predictions...". Gavrilakis (1992) concluded that "a theory on the secondary flow structures that give rise to the observed mean flow is not yet available".

Einstein (1942) postulated that a channel flow could be divided into three subflow regions corresponding to the sidewalls and bed; thus, the turbulent characteristics of flow in each sub-region would be dominated by the contiguous solid boundary. However, Einstein did not illustrate why the flow region is dividable and how to divide the flow area. Keulegan (1938) also touched upon the need to separate the flow cross-sectional area into different regions; he suggested that
Fig. 1. Definition and sketch for a rectangular duct flow.

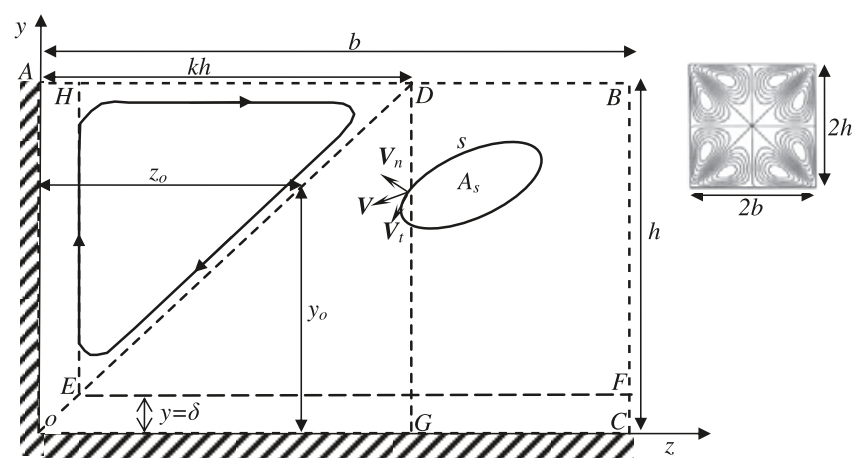

the bisectors of base angles of the channel could be used to divide the flow region because the flow characteristics in regions separated by it are different from each other. The bisectors have been widely used to separate two conjoining secondary currents (Aly et al. 1978). Chien and Wan (1999) concisely explained the physical meaning of Einstein's idea in terms of how the turbulent energy should be transferred and eventually dissipated as heat at the boundary (Yang et al. 2005). They asserted that the turbulent energy in any unit flow volume will be transported toward either sidewalls or bed to be dissipated. Furthermore, Yang (1993) and Yang and Lim (1997) proposed that the turbulent energy is always transferred through a minimum relative distance toward the nearest boundary to be dissipated, where the relative distance is defined as the ratio of the normal distance between the location of the energy source concerned and the boundary to the turbulent energy dissipation capacity of the boundary, $D$ (Lim and Yang 2005). For a smooth boundary, $D$ is the viscous length scale $\left(=v / u_{*}\right)$, where $v$ is the kinematic viscosity of fluid, and $u *$ is the shear velocity. For a rough boundary, $D$ is scaled using the boundary roughness height, or $D=\Delta$.

The main objectives of this study are to (i) identify the region and shape of secondary currents in a duct flow; (ii) investigate the underlying mechanism of secondary currents by following the line of concept about the turbulent energy transport; and (iii) establish a relation between the near-wall strength of secondary currents and the lateral gradient of streamwise velocity.

\section{Relation of total shear stress and velocity normal to a curve}

This study focuses on the steady, uniform, and fully developed turbulent duct flow with arbitrary aspect ratio $b / h$, bed width of $2 b$, and height of $2 h$. O Figure 1 shows one quarter of the cross section. The flow in a control volume bounded by a simple closed curve " $s$ " is first discussed: the normal unit vector of " $s$ " is defined as $\boldsymbol{n}$, the instantaneous velocity in the streamwise direction is $U$, and the instantaneous velocity in $y z$-plane is defined as vector $\boldsymbol{V}$.

The total shear stress on curve s can be defined as (Yang et al. 2004; Yang and Lim 2006)

$$
\text { [1] } \quad \tau=\mu \frac{\partial \bar{U}}{\partial n}-\rho \overline{U V_{n}}
$$

where $\mu$ is the dynamic viscosity, $\rho$ is the fluid density, $\tau$ is 
the total shear stress on the interface of $s, \partial \bar{U} / \partial n$ is the velocity gradient in the $\boldsymbol{n}$ direction, where the overbar denotes the time-averaged values, and

[2] $\quad \boldsymbol{V}=V_{n} \boldsymbol{e}_{n}+V_{t} \boldsymbol{e}_{t}$

where $V_{n}$ is the component of $\boldsymbol{V}$ in the direction of $\boldsymbol{n}$, and $V_{t}$ is the component of $\boldsymbol{V}$ in the tangent direction, and $\boldsymbol{e}$ is a unit vector. Therefore, the force balance in $x$-direction can be expressed as

[3] $\oint_{s} \tau \mathrm{d} s=\rho g S A_{\mathrm{s}}=\iint_{A_{s}} \rho g S \mathrm{~d} y \mathrm{~d} z$

where $S$ is the energy slope and $A_{s}$ is the area bounded by "s".

[4] $\oint_{s} \frac{\partial \bar{U}}{\partial n} \mathrm{~d} s=\oint_{s}\left(\frac{\partial u}{\partial y} \vec{j}+\frac{\partial u}{\partial z} \vec{k}\right) \bullet\left(\frac{\mathrm{d} z}{\mathrm{~d} s} \vec{j}-\frac{\mathrm{d} y}{\mathrm{~d} s} \vec{k}\right) \mathrm{d} s=\oint_{s}\left(\frac{\partial u}{\partial y} \mathrm{~d} z-\frac{\partial u}{\partial z} \mathrm{~d} y\right)$

where $j$ and $k$ are unit vectors in $y$ and $z$ directions, respectively, here $U=u+u^{\prime}$ is introduced, $u$ is the time-averaged velocity in $x$ direction.

By applying Green's theorem to eq. [4], one obtains

$[5] \quad \oint_{s} \frac{\partial \bar{U}}{\partial n} \mathrm{~d} s=\iint_{A_{s}}-\left(\frac{\partial^{2} u}{\partial z^{2}}+\frac{\partial^{2} u}{\partial y^{2}}\right) \mathrm{d} y \mathrm{~d} z$

where $v$ and $w$ represent time-average velocities in $y$ and $z$ directions, respectively; and $u^{\prime}, v^{\prime}$, and $w^{\prime}$ are turbulent velocity fluctuations. Therefore, the following relation can be derived

[6] $\quad V_{n}=\vec{V} \bullet \vec{n}=\left[\left(v+v^{\prime}\right) \vec{j}+\left(w+w^{\prime}\right) \vec{k}\right] \bullet\left(\frac{\mathrm{d} z}{\mathrm{~d} s} \vec{j}-\frac{\mathrm{d} y}{\mathrm{~d} s} \vec{k}\right)=\left(v+v^{\prime}\right) \frac{\mathrm{d} z}{\mathrm{~d} s}-\left(w+w^{\prime}\right) \frac{\mathrm{d} y}{\mathrm{~d} s}$

[7] $\oint_{s} \overline{U V}_{n} \mathrm{~d} s=\oint_{\mathrm{s}} u(v \mathrm{~d} z-w \mathrm{~d} y)+\oint_{s}\left(\overline{u^{\prime} w^{\prime}} \mathrm{d} z-\overline{u^{\prime} w^{\prime}} \mathrm{d} y\right)$

using eqs. [3], [5], and [7], one obtains

[8] $\oint_{s} \tau \mathrm{d} s=\oint_{s} \rho u(w \mathrm{~d} y-v \mathrm{~d} z)+\oint_{s}\left(\tau_{\mathrm{xy}} \mathrm{d} z-\tau_{\mathrm{xz}} \mathrm{d} y\right)$

where $\tau_{\mathrm{xy}}=\mu \partial u / \partial y-\rho \overline{u^{\prime} v^{\prime}}$ and $\tau_{\mathrm{xz}}=\mu \partial u / \partial z-\rho \overline{u^{\prime} w^{\prime}}$.

By applying Green's theorem to eq. [8], one gets

[9] $\oint_{s} \tau \mathrm{d} s=\iint_{A_{s}}\left[\frac{\partial\left(\rho u v-\tau_{\mathrm{xy}}\right)}{\partial y}+\frac{\partial\left(\rho u w-\tau_{\mathrm{xz}}\right)}{\partial z}\right] \mathrm{d} y \mathrm{~d} z$

the continuity equation is given as

[10] $\frac{\partial v}{\partial y}+\frac{\partial w}{\partial z}=0$

inserting eq. [9] into eq. [3], one obtains the following equation

$$
\frac{\partial\left(u v-\tau_{\mathrm{xy}} / \rho\right)}{\partial y}+\frac{\partial\left(u w-\tau_{\mathrm{xz}} / \rho\right)}{\partial z}=g S
$$

by subtracting eq. [10] from eq. [11], one obtains the conventional Reynolds equation. This indicates that the shear stress defined in eq. [1] is correct.

From eqs. [1] and [11], one can conclude that the total shear stress on an arbitrary interface is the time-averaged product of instantaneous velocity $U$ and $V_{n}$.

The conventional expression of shear stress includes two parts, one is the viscous shear stress and the other Reynolds shear stress, in the main flow region, the viscous shear stress is negligible relative to the Reynolds shear stress.
As $U=u+u^{\prime}$ and $V_{n}=v_{n}+v_{n}^{\prime}$, the total shear stress in eq. [1] can be decomposed into two components follows:

$$
-\overline{U V_{n}}=-u v_{n}-{\overline{u^{\prime} w^{\prime}}}_{n}
$$

The first term on the right-hand side (RHS) of eq. [12] is the shear stress caused by the mean flow, and the second term is the Reynolds shear stress induced by the velocity fluctuations. The significance of eq. [12] is that $u v_{n}$ is also a type of shear stress, just similar to the Reynolds shear stress. With the presence of secondary currents, obviously the first term or $u v_{n}$ is nonzero. It would be interesting to investigate how the nonzero velocity $v_{n}$ is formed and what is the relation between $v_{n}$ and secondary currents.

Mathmatically, it can be inferred from eq. [1] that if the flow region is divisible as postulated by Einstein (1942), its total shear stress along division line must be zero or the following conditions must be satisfied:

(i) as $v_{\mathrm{o}} \mathrm{d} z_{\mathrm{o}}-w_{\mathrm{o}} \mathrm{d} y_{\mathrm{o}}=0$, this requires

[13a] $\frac{w_{\mathrm{o}}}{v_{\mathrm{o}}}=\frac{\mathrm{d} z_{\mathrm{o}}}{\mathrm{d} y_{\mathrm{o}}}=k$

where $\mathrm{d} z_{\mathrm{o}} / \mathrm{d} y_{\mathrm{o}}$ is the gradient of the division line, the gradient of bisector is $\mathrm{d} z_{\mathrm{o}} / \mathrm{d} y_{\mathrm{o}}=1 ; w_{\mathrm{o}} / v_{\mathrm{o}}$ is the ratio of the components of secondary current along the division line. Physically, this means that $\left(v_{\mathrm{o}} \boldsymbol{j}+w_{\mathrm{o}} \cdot \boldsymbol{k}\right)\left(\mathrm{d} z_{\mathrm{o}} \boldsymbol{j} \mathrm{d} y_{\mathrm{o}} \boldsymbol{k}\right)=0$ or $V_{n}=0$.

In other words, the division line must be the interface of neighbouring secondary cells: (ii) along the division line, the Reynolds shear stress should satisfy: $\tau_{\mathrm{yx}} \mathrm{d} z_{\mathrm{o}}-\tau_{z x} \mathrm{~d} y_{\mathrm{o}}=0$, where $\mathrm{d} z_{\mathrm{o}} / \mathrm{d} y_{\mathrm{o}}$ is also the gradient of division line, or

$$
\left(-\overline{u^{\prime} w^{\prime}}\right)_{\mathrm{o}}=k\left(-\overline{u^{\prime} v^{\prime}}\right)_{\mathrm{o}}
$$

Physically, this means that there exists no exchange of fluid particles across the division line driven by mean flow. 
This is consistent with Gessner's (1973) experimental conclusion.

It is obvious that the symmetrical lines of the channel (e.g., "AB" and "BC" in Fig. 1) satisfy the above two conditions, this means that the symmetrical lines can be used as division lines because eq. [8] indicates that the total shear stress is zero along these lines.

\section{Shape of a secondary cell}

Prandtl's (1926) and Keulegan (1938) postulated that in ducts and open channels, the flow region can be separated by the bisectors of base angle (Schlichting 1979). Yang and his co-workers (Yang and Lim 1997; Yang and McCorquodale 2004; Lim and Yang 2005) proposed that the solid boundary always dissipates the turbulent energy from the contiguous flow body or the turbulent energy received from the main flow is transported through a minimum relative distance toward a unit area on the wetted perimeter. This leads to the following equation for the division line

$$
\frac{y_{\mathrm{o}}}{\nu / \bar{u}_{* \mathrm{~b}}}=\frac{z_{\mathrm{o}}}{v / \bar{u}_{* \mathrm{w}}} \quad \text { or } \quad k=\frac{z}{y}=\frac{\bar{u}_{* \mathrm{~b}}}{\bar{u}_{* \mathrm{w}}}
$$

where $\bar{u}_{* \mathrm{~b}}=\sqrt{\bar{\tau}_{\mathrm{b}}} / \rho ; \bar{u}_{* \mathrm{w}}=\sqrt{\bar{\tau}_{\mathrm{w}}} / \rho ; u_{*}$ is the shear velocity; and the subscripts $\mathrm{b}$ and $\mathrm{w}$ denote the bed and wall, respectively.

In eq. [14], $y /\left(v / \bar{u}_{* \mathrm{~b}}\right)$ is the relative distance to the bed for any point in the flow field, and $z /\left(v / \bar{u}_{* \mathrm{w}}\right)$ is the relative distance from the point to the sidewall. All points in region oDBC of Fig. 1 satisfy $y /\left(v / \bar{u}_{* \mathrm{~b}}\right)<z /\left(v / \bar{u}_{* \mathrm{w}}\right)$, thus the turbulent energy in this region will be dissipated on the bed oC. Likewise, the turbulent energy contained in the volume of oAD will be dissipated on the wall oA. In other words, the turbulent characteristics in regions $\mathrm{OAD}$ and $\mathrm{oDBC}$ are independent of each other, they are governed individually by the sidewall and bed, respectively, as presumed by Einstein.

The area $\mathrm{OAD}$ is $k h^{2} / 2$, the mean wall shear stresses along $\mathrm{oA}$ is $\bar{\tau}_{\mathrm{w}}$, and the total shear stress along $\mathrm{AD}$ and $\mathrm{oD}$ are zero because no particle crosses these lines to exchange their momentum, therefore according to eq. [3], one can determine the mean sidewall shear stress by

[15] $\quad \bar{\tau}_{\mathrm{w}} h=\rho g S \frac{k h^{2}}{2} \quad$ or $\quad \frac{\bar{\tau}_{\mathrm{w}}}{\rho g h S}=\frac{k}{2}$

Similarly, the mean bed shear stress along oC is $\bar{\tau}_{\mathrm{b}}$ but $\tau$ is zero along the enclosed curve oDBC, then eq. [3] gives

[16] $\quad \bar{\tau}_{\mathrm{b}} b=\rho g S \frac{b+(b-k h)}{2} \quad$ or $\quad \frac{\bar{\tau}_{\mathrm{b}}}{\rho g h S}=1-\frac{k}{2} \frac{h}{b}$

Using $\bar{\tau}_{\mathrm{b}}=\rho \bar{u}_{* \mathrm{~b}}^{2}$ and $\bar{\tau}_{\mathrm{w}}=\rho \bar{u}_{* \mathrm{w}}^{2}$, one obtains the following equation by inserting eqs. [15] and [16] into eq. [14]

[17] $\quad k^{3}+k \frac{h}{b}-2=0$

Equation [17] indicates that $k=1$, if $b / h=1$; and if a channel is shallow-wide, i.e., $b / h \rightarrow \infty, k$ approaches to $\sqrt[3]{2}=1.26$; this means that the pair of secondary currents can be separated by the line of $y_{0}=1.26 z_{0}$, this conclusion is different from previous postulations by Prandtl (1926),
Fig. 2. Measured Reynolds shear stress distribution along vertical profiles in a rectangular duct (Tracy 1965), the solid symbols indicate that in the central region $(z / h>1.2)$, the Reynolds shear stress follows the linear relationship, but in the near-sidewall region, the data points deviate from the straight line, thereby implying the existence of secondary currents.

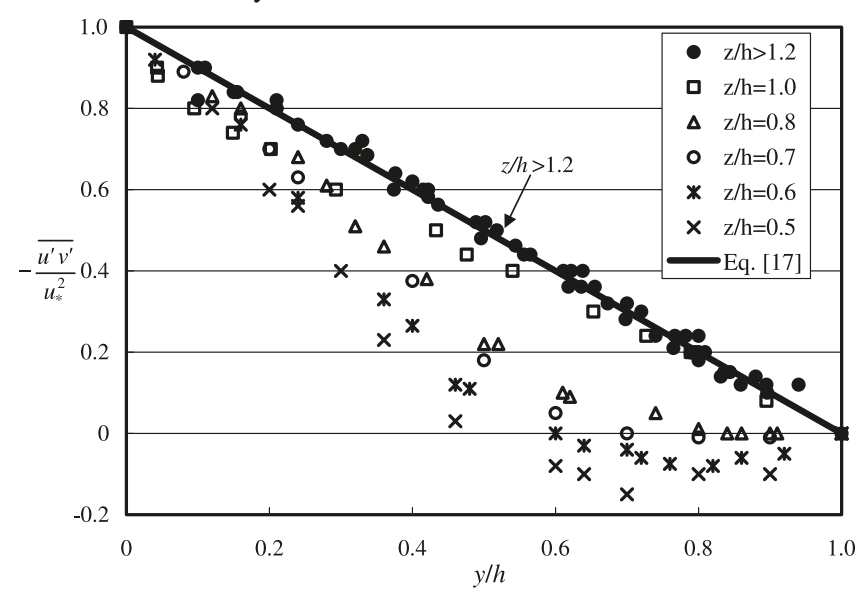

Keulegan (1938), Einsten (1942), Schlichting (1979), etc. Therefore, it would be useful to examine the division line or to check the existence of critical value 1.26 using experimental data.

Tracy (1965) measured the secondary currents in a wide smooth rectangular wind tunnel $(b / h=6.4)$ using a constant-current Thiele Wright hot-wire anemometer, his results show that the turbulent structures, e.g., Reynolds shear stress and turbulence fluctuations in the region of GDBC are totally different from that in the region of oDG. The measured Reynolds shear stress profiles are reproduced in Fig. 2, it can be seen clearly that in GDBC $(z / h>1.2)$ is independent of $z$ and follows the standard linear line:

$$
-\frac{\overline{u^{\prime} v^{\prime}}}{u_{*}^{2}}=1-\frac{y}{h}
$$

but for profiles in the region oDG where $z<1.2 h$, the Reynolds shear stress deviates from eq. [18]. Hence, one can conclude that there indeed exists the critical value of $k=1.2$.

To check the existence of critical value 1.26 , the measured mean wall and bed shear stresses of Knight and Patel (1985), and Rhodes and Knight (1994) are used to analyze. The datasets cover a wide range of $b / h$ from 0.01 to 50. Figures $3 a$ and $3 b$ show comparisons between measured and computed $\bar{\tau}_{\mathrm{w}} / \rho g h S$ and $\bar{\tau}_{\mathrm{b}} / \rho g h S$ plotted against aspect ratio $b / h$, which include eqs. [15] and [16] and for a given aspect ratio the value of $k$ can be determined from eq. [17].

It is interesting to note that in Fig. $3 a$ the measured $\bar{\tau}_{\mathrm{w}} / \rho g h S$ is constant and equal to 0.63 for $b / h>20$, which is precisely half of the critical value 1.26 discovered from Tracy's experiment. As the experiment results can be interpreted as $\bar{\tau}_{\mathrm{w}} h=\rho g S A_{s}$ and the area where energy is dissipated on the wall is $A_{s}=1.26 h^{2} / 2$, it can be impliedd that the area is a triangle. Hence, different experimenters observed the existence of critical value of 1.26 using different methods, which indicates that the width of region affected 
Fig. 3. Comparison between $\bar{\tau}_{\mathrm{w}} / \rho g h S$ and $\bar{\tau}_{\mathrm{b}} / \rho g h S$ computed and measured data.

(a)

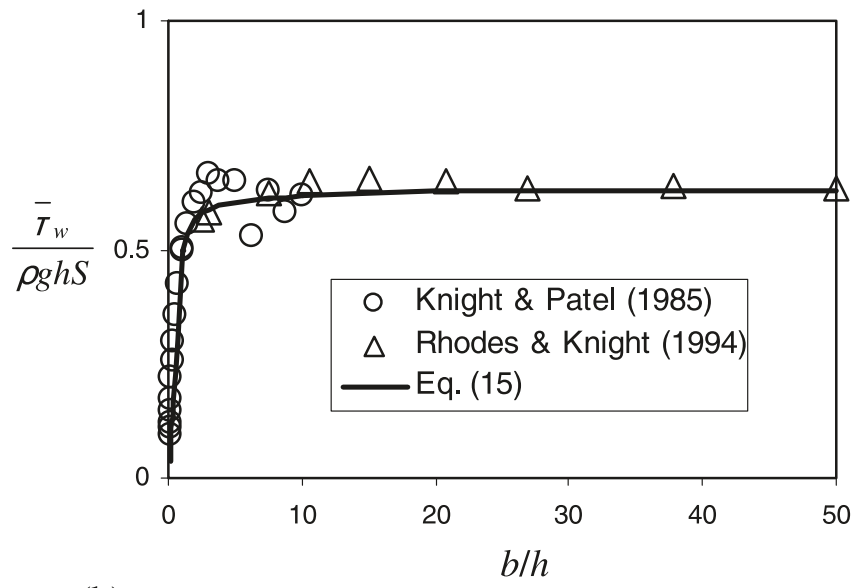

(b)

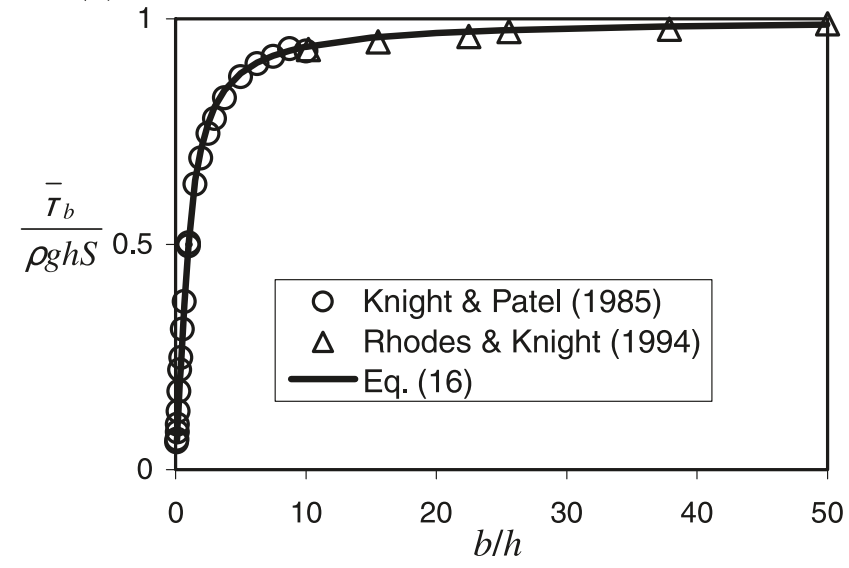

by sidewall area is up to $1.26 h$, rather than $1.0 h$ assumed by previous researchers.

Therefore, it can be concluded that no mean flow penetrates the symmetrical lines $\mathrm{AB}$ and $\mathrm{BC}$ in Fig. 1 as well as the division line $\mathrm{oD}$, thus the secondary currents will be constrained to move inside the domains of $\mathrm{OAD}$ and oDBC. This actually identifies the shapes of secondary currents.

\section{Reynolds shear stress distribution}

The experimental researchers, such as Nezu and Nakagawa (1993), Imamoto and Ishigaki (1988), and Tracy (1965), observed that the value of $-\overline{u^{\prime} v^{\prime}}$ maintains a linear distribution along the normal line of boundary from the bed to division lines. This phenomenon is consistent with the concept that the turbulent energy is transferred along the minimum relative distance, thus the Reynolds shear stress distribution can be written as follows:

Along a vertical line in oDBC

$$
\frac{\partial \tau_{x y}}{\partial y}=-\rho g S_{1(z)}
$$

Along a horizontal line in $\mathrm{OAD}$

$$
\frac{\partial \tau_{x z}}{\partial z}=-\rho g S_{2(y)}
$$

where $\rho g S_{1}$ and $\rho g S_{2}$ are gradients of the measured Reynolds shear stress in $y$ and $z$ directions, respectively.

Integration of eq. [19] with respect to $y$ from the bed to the upper boundary oDB yields

$$
\frac{-\overline{u^{\prime} v^{\prime}}}{u_{* \mathrm{~b}(z)}^{2}}=\left(1-\frac{y}{y_{\mathrm{o}}}\right)+\frac{\left(-\overline{u^{\prime} v^{\prime}}\right)_{\mathrm{o}}}{u_{* \mathrm{~b}(z)}^{2}}
$$

where $y_{0}$ is the distance from the bed to the division lines, $\left(-\overline{u^{\prime} v^{\prime}}\right)_{\mathrm{o}}$ is the Reynolds shear stress on the division lines, and shear stress at the local boundary $\tau_{\mathrm{b}(z)}$ can be determined by

$$
\tau_{\mathrm{b}(z)}=\rho u_{* \mathrm{~b}(z)}^{2}=\rho g S_{1} y_{\mathrm{o}}+\rho\left(-\overline{u^{\prime} v^{\prime}}\right)_{\mathrm{o}}
$$

Similarly, in the region oAD the Reynolds shear stress distribution can be obtained by integrating eq. [20] with respect to $z$

$$
\begin{aligned}
& \frac{-\overline{u^{\prime} w^{\prime}}}{u_{* \mathrm{w}(y)}^{2}}=\left(1-\frac{z}{z_{\mathrm{o}}}\right)+\frac{\left(-\overline{u^{\prime} w^{\prime}}\right)_{\mathrm{o}}}{u_{* \mathrm{w}(y)}^{2}} \\
& \tau_{\mathrm{w}(\mathrm{y})}=\rho u_{* \mathrm{w}(y)}^{2}=\rho g S_{2} z_{\mathrm{o}}+\rho\left(-\overline{u^{\prime} w^{\prime}}\right)_{\mathrm{o}}
\end{aligned}
$$

Using eqs. [21] and [23], one can assess $S_{1}$ and $S_{2}$ based on measured Reynolds shear stress. In this study, the experimental data by Sata et al. (1994) are used for estimating $S_{1}$ and $S_{2}$. A three-dimensional (3-D) particle tracking velocimeter was applied to an air flow in a square cross-sectional duct. The cross section of the duct was $71 \mathrm{~mm} \times 71 \mathrm{~mm}$, and a test section was located 70 times the hydraulic diameter downstream from an inlet of the duct. A measurement volume was $1 / 4$ of the cross section that includes the corner between the side and bottom walls. Cross-stream distributions of the mean velocities and of the full Reynolds stress tensor components were obtained and some of them were compared with a separate hot-wire measurement. The agreement was generally good for both methods, indicating the datasets could be reliable. The measured data are available in http://www.ted-jsme.jp/HTDB/fw.html.

For a square duct $(b / h=1)$, eq. [17] gives $k=1$, thus the division line is identical to the bisector or no mean flow penetrates the bisector. The typical Reynolds shear stress distribution along the same path of energy transport is shown in Fig. 4, in which the shear velocity $u_{*}$ is determined by extrapolating the measured Reynolds shear stress to the bed. Using eq. [22], $S_{1}$ can be determined based on $u_{*}$ and $\left(-\overline{u^{\prime} v^{\prime}}\right)_{\mathrm{o}}$.

Equation [23] clearly shows that along the bed normal line, the Reynolds shear stress increases linearly with $y / h$ from the bed to the division line, but the slope of these straight lines is variable, thus it is necessary to investigate $S_{1}$ in eq. [19]. Tracy's measurement shows that in the region of DGCB, $S_{1}=S$, whereas in the region of oDG the slopes in profiles are different from each other, the constraint condition for $S_{1}$ can be obtained from eqs. [15] and [16] by using 
Fig. 4. Profiles of Reynolds shear stress $\left(-\overline{u^{\prime} w^{\prime}}+\overline{u^{\prime} w^{\prime}}{ }_{\mathrm{o}}\right) / u_{* \mathrm{~b}(z)}^{2}$ in a square duct flow based on the data by Sata et al. (1994).

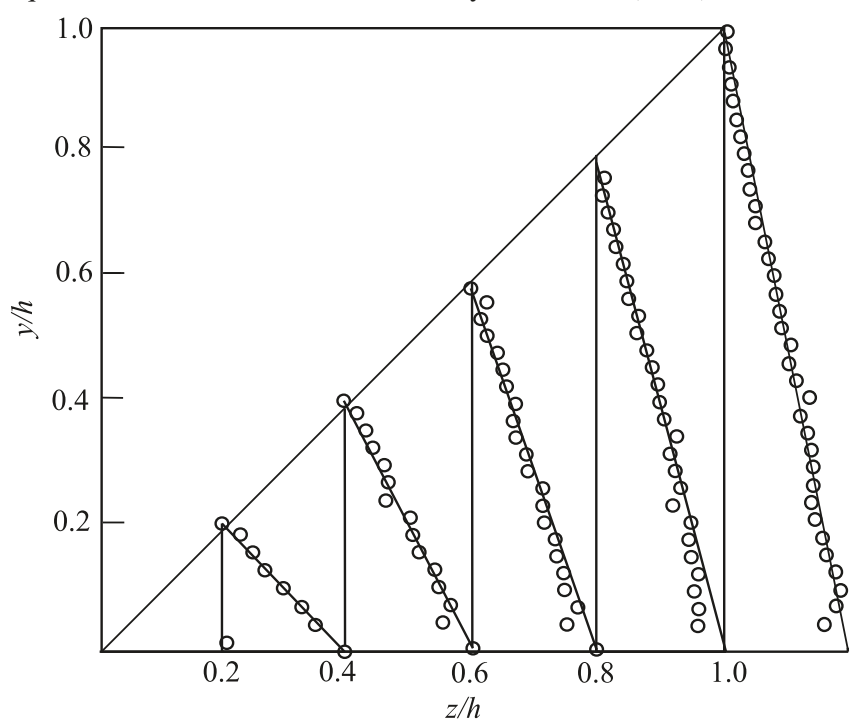

the definition of $\bar{\tau}_{\mathrm{b}}=\int_{0}^{b} \tau_{\mathrm{b}(z)} \mathrm{d} z / b$ and $\bar{\tau}_{\mathrm{w}}=\int_{0}^{b} \tau_{\mathrm{w}(y)} \mathrm{d} y / h$. Therefore, one obtains

$$
\int_{0}^{1} \frac{S_{1}}{S} z^{\prime} \mathrm{d} z^{\prime}+\int_{0}^{1} \frac{\left(-\overline{u^{\prime} v^{\prime}}\right)_{\mathrm{o}}}{g k h S} \mathrm{~d} z^{\prime}=\frac{1}{2}
$$

$$
\int_{0}^{1} \frac{S_{2}}{S} y^{\prime} \mathrm{d} y^{\prime}+\int_{0}^{1} \frac{\left(-\overline{u^{\prime} w^{\prime}}\right)_{\mathrm{o}}}{g h S} \mathrm{~d} y^{\prime}=\frac{1}{2}
$$

where $z^{\prime}=z /(k h)$ and $y^{\prime}=y / h$.

Obviously, if $S=S_{1}=S_{2}$, then $\left(-\overline{u^{\prime} v^{\prime}}\right)_{\mathrm{o}}=\left(-\overline{u^{\prime} w^{\prime}}\right)_{\mathrm{o}}=0$. Yang and Lim (1997) and Lim and Yang (2005) obtained the distribution of local boundary shear stress based on such assumption, their simplified model shows that the local boundary shear stress distribution is linear in the corner region, which is slightly different from the measurements by Knight et al. (1984) and Knight and Patel (1985), thus the error source of their model has been identified, i.e., $S_{1} / S \neq 1$ and $S_{2} / S \neq 1$. The experimental data shown in Fig. 5 by Sata et al. (1994) clearly indicate that $S_{1}$ is a variable.

\section{Underlying mechanism of secondary currents}

The near-bed region oEFC in Fig. 1 is taken into consideration, where the secondary flow is parallel to the bed or the wall-normal velocity is zero, thus in eq. [11] the term of $u v$ is negligible relative to $\tau_{x y} / \rho\left(\approx u_{*}^{2}\right)$. Based on the fact that the shear stress distribution is linear along the same path of turbulent energy transport, i.e., eq. [19], one can obtain the governing equations for the secondary currents from eq. [11] as follows:

For the region of oEFC

$$
\frac{\partial\left(u w-\tau_{x z} / \rho\right)}{\partial z}=g\left(S-S_{1}\right)
$$

Similarly, for the region very near the sidewall, say oEHA,
Fig. 5. Variation of $S_{1} / S$ in a square duct flow, data derived from measured Reynolds shear stress.

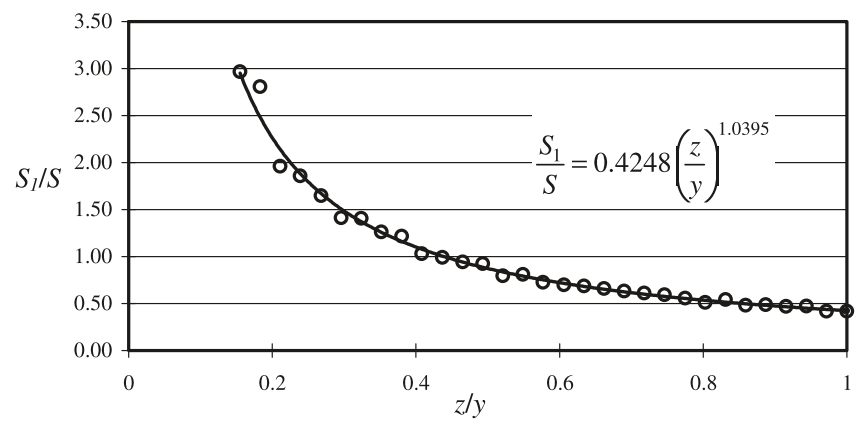

the secondary flow is parallel to the sidewall, and the term of $u w$ in eq. [11] is negligible relative to $\tau_{x z} / \rho\left(=u_{* y}^{2}\right)$. Equation [11] can be simplified as follows using eq. [20]

For the region oEHA

$$
\frac{\partial\left(u v-\tau_{\mathrm{xy}} / \rho\right)}{\partial y}=g\left(S-S_{2}\right)
$$

the integration of eq. [27] with respect to $z$ along the line CE yields

$$
\frac{u w}{g k h S}=\frac{-\overline{u^{\prime} w^{\prime}}}{g k h S}+\int_{\delta^{\prime}}^{z^{\prime}}\left(1-\frac{S_{1}}{S}\right) \mathrm{d} z^{\prime}
$$

where $z^{\prime}=z / h \delta^{\prime}=\delta / h$, and $\delta$ is the thickness of viscous sublayer, and $\delta / h \approx 0$.

The integration of eq. [28] with respect to $y$ along the line of EH yields

$$
\frac{u v}{g h S}=\frac{-\overline{u^{\prime} v^{\prime}}}{g h S}+\int_{\delta^{\prime}}^{y^{\prime}}\left(1-\frac{S_{2}}{S}\right) \mathrm{d} y^{\prime}
$$

where $y^{\prime}=y / h$.

Equations [29] and [30] show that the amplitude of nearwall secondary currents ( $w$ or $v$ ) can be fully determined by the mean velocity, $u$, Reynolds shear stress(Fig. 6), as well as its distribution. This is different from previous hypotheses made by Einstein and Li (1958) because it either explicitly or implicitly, overstates the importance of normal Reynolds stress, which differs from the Reynolds shear stress that is considered constant near the wall. Figure 7 shows the plot of secondary flow $(u w / g h S)$ versus the near-wall Reynolds shear stress and its distribution based on the measured data by Sata et al. (1994), in which the regression equation shown in Fig. 5 is included in the calculation for $S_{1} / S$. It can be seen clearly that data from Sata et al. (1994) support eq. [29]. The scatter of data points is caused by the measurement error. As stated by the experimenters, the measurement error in the near-wall region was relatively high.

Equations [29] and [30] highlight the importance of Reynolds shear stress that can be related to the streamwise velocity using Boussinesq's (Boussinesq 1877) equation

$$
\text { [31] }-\overline{u^{\prime} w^{\prime}}=v_{\mathrm{t}} \frac{\mathrm{d} u}{\mathrm{~d} z}
$$

where $v_{\mathrm{t}}$ is the transverse eddy viscosity. 
Fig. 6. Reynolds shear stress along a division measured by Sata et al. (1994).

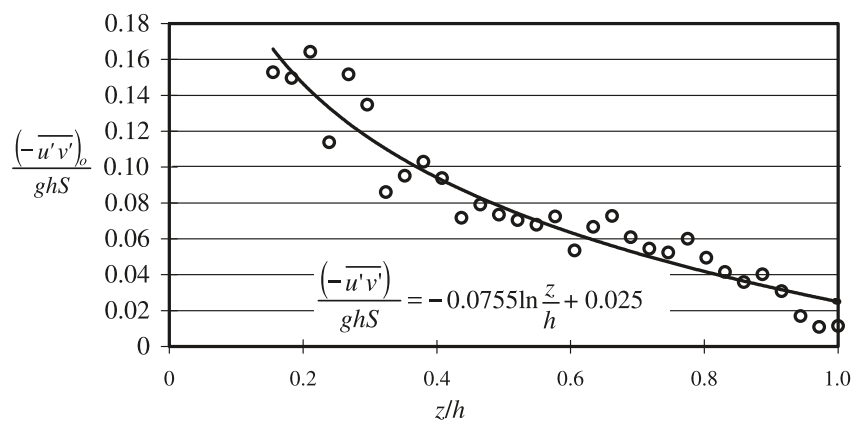

Fig. 7. Relation between the near-wall strength of secondary flow and the near-wall Reynolds shear stress and its distribution.

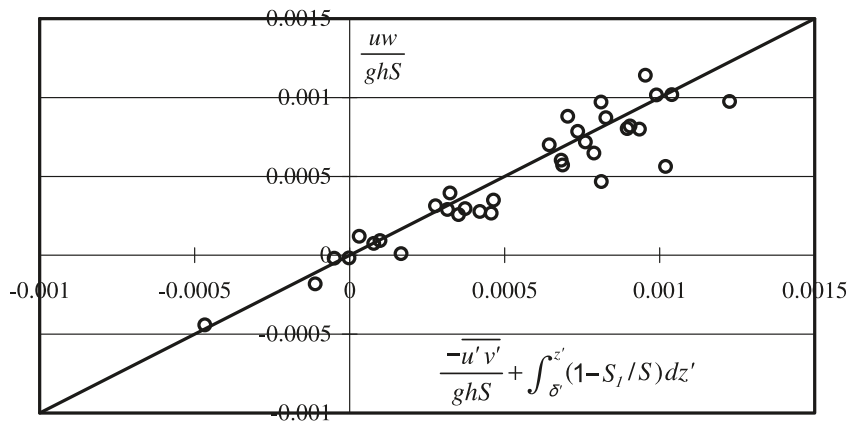

Substituting eq. [31] into eq. [29], one obtains the following expression of velocity $w$,

$$
\frac{u w}{g k h S}=\frac{v_{\mathrm{t}}}{g k h S} \frac{\partial u}{\partial z}+\int_{\delta \prime}^{z^{\prime}}\left(1-\frac{S_{1}}{S}\right) \mathrm{d} z^{\prime}
$$

Equation [32] demonstrates that the secondary flow exists because of the nonzero wall-tangent velocity gradient, i.e., $\partial u / \partial z$. In other words, one can say that no secondary currents will be observed if in the near-bed region $\partial u / \partial z=0$ and $S_{1}=S$. This premise has been confirmed by Nezu et al. (1985) experimentally in wide channels, where no secondary currents were detected in its central zone of very wide channel. Hence, this study indicates that the source of secondary currents comes from the boundary. It is well known that the solid wall is responsible for the generation of turbulence or small-scale eddies driven by the wall-normal gradient of near-bed velocity, $\partial u / \partial y$; and the velocity variation in the flow direction yields flow separation, i.e., horseshoe eddies behind bridge piers, or Karman vortex street. Equation [32] states that the solid wall is also responsible for the generation of secondary currents, if the wall-tangent velocity gradient $\partial u / \partial z$ is nonzero. Therefore, we can conclude that all eddies comes for the variation of near boundary velocity, no matter how small or big its sizes are, the near wall region is the source of all vortices, its orientation depends on the velocity gradient in $x, y$, and $z$ direction.

The above discussion demonstrates the relationship between the wall-tangent velocity $w$ and $\partial u / \partial z$. The next question that has to be answered clearly is how the near-bed velocity $w$ induces the secondary currents, or alternatively one has to clarify whether the nonzero wall-tangent velocity
Fig. 8. Recirculating flow in cavities induced by nonzero velocity $w$. (a)

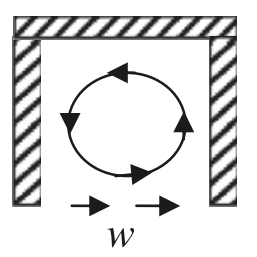

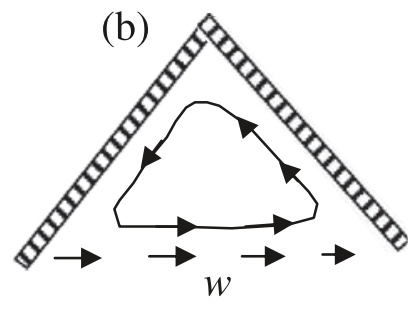

$w$ certainly generates secondary currents. Equation [32] shows that this is the only element responsible for generating secondary currents.

As mentioned earlier, no mean flow penetrates division lines, thus the wall-tangent flow $w$ has to change its direction when it encounters these interfaces with zero total shear stress because the continuity equation has to be satisfied. Therefore, the flow in the plane perpendicular to the main streamwise direction has to be circulated or the vortex is formed. The mechanism is very similar to cases shown in Fig. 8 in which the vortices have been observed widely in field and laboratory (Liu 2006; Hangen and Dhanak 1966; van Schijndel 1998; Schmidt 1990). In the experiment by Liu (2006), the nonzero velocity $w$ was artificially generated and then, the circulation shown in Fig. $8 a$ became discernible. Similarly, the velocity $w$ can drive the vortex in Fig. $8 b$, which indicates that the division lines in Fig. 1 and the solid walls in Fig. 8 are functionally identical because no mean flow penetrates these lines, and these lines confine the induced recirculating flow. Hence, one can conclude that the nonzero velocity $w$ generates the secondary currents.

The importance of solid boundary has been known because the kinetic energy is converted (transformed) into thermal energy and ultimately dissipated by viscous effect in the near-wall region, however, the importance of solid boundary for the generation of secondary currents have long been underestimated or ignored by almost all other researchers. Equation [32] and Fig. 8 demonstrate that the sufficient prerequisite for the formation of secondary currents is the nonzero wall-tangent velocity $w$, induced by near-bed $\partial u / \partial z$. It is well known that for a smooth rectangular channel, the near-bed velocity $u$ is proportional to the shear velocity $u_{*(\mathrm{z})}$, which is closely related to the Reynolds shear stress. Hence, eq. [32] can be interpreted as the mechanism for initiating secondary currents, when the driving force is caused by the lateral gradient of Reynolds shear stress, which is in line with the arguments by Gessner (1973).

To show that the secondary flow is indeed produced by the lateral variation of near-bed velocity, the experimental results shown in Fig. 9 are discussed. Nezu and Nakagawa (1993; p. 118) measured the secondary currents using Xtype hot-wire anamometers in a uniform flow, artificial longitudinal ridge elements were attached to the smooth bed. The spanwise spacing of ridges was chosen same as the water depth, a pair of longitudinal vortices were confined to the region between two ridges. The measured results are reproduced in Fig. 9, which shows that a strong cellular currents appeared on either side of the ridge as the result of small disturbance of nonzero $\partial u / \partial z$ and the secondary flow moves to the ridge where the velocity is higher. The experi- 
Fig. 9. Secondary currents over artificial ridges in a uniform flow measured by Nezu and Nakagawa (1993).

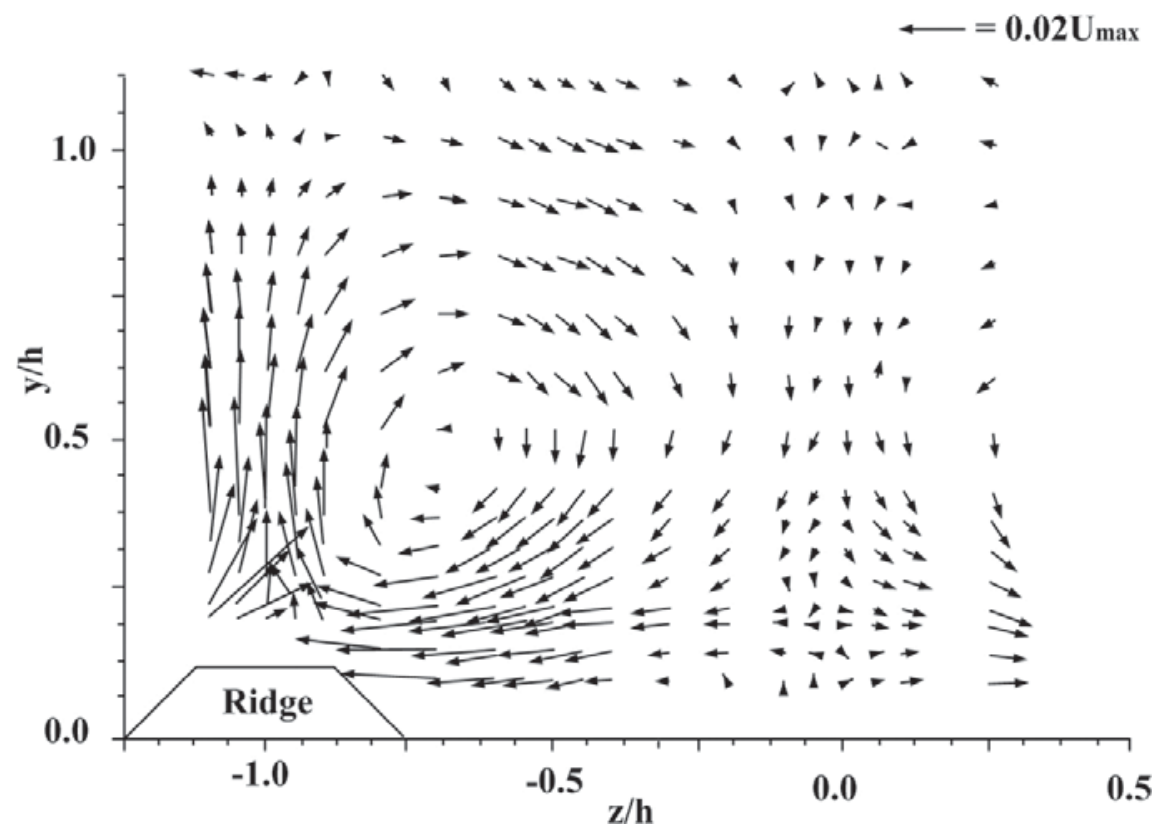

ments by Nezu and Nakagawa (1984) obviously confirm that the secondary currents are always associated with or occur at the place where the near-bed regional velocity, $\partial u / \partial z$ $\neq 0$. It can be predicted that no secondary currents would be induced if the ridge was placed on the upper flow region, instead of the bed. This means that the initiation mechanism of secondary currents is the lateral variation of near-bed velocity. In fact, Nezu et al. (1985) also speculated the significance of near-bed velocity for the initiation mechanism of secondary currents as they commented that "small disturbances present on the bed may provide driving forces that generate or enhance secondary motions".

The lateral variation of streamwise velocity $(\partial u / \partial z \neq 0)$, caused by nonuniform boundary shear stress $\left(S_{1} / S \neq 0\right)$ or a ridge shown in Fig. $9 a$ and $9 b$, respectively, leads to the presence of wall-tangent velocity, then the wall-normal mean flows will be induced because the continuity equation, i.e., eq. [10] must be satisfied. Hence, it can be inferred that the secondary currents would have the following characteristics:

- The secondary currents will be generated if $\partial u / \partial z \neq 0$ or sufficient condition for the existence of secondary currents is the lateral variation of near-bed velocity.

- The secondary currents will never penetrate division lines. Thus, lines with zero shear stress $\tau$ delineate the size and shape of secondary currents.

\section{Features of secondary currents}

Now, it is difficult to obtain the analytical solution of eq. [32] because it involves the distribution of near-bed velocity distribution and Reynolds shear stress, which can be only predicted using mathematical models. But numerical models, regardless of the degree of complexity, require much more effort and time than an ordinary engineer could provide for simple case-flow in a rectangular, straight duct. Thus, estimation of important features of secondary currents, such as the locations of vortex center, the size of forced vortex, and the near-bed secondary velocity, may be useful in practice.

Based on the aforementioned discussions, the vortex center in the $\mathrm{OAD}$ region could be assumed to be identical with the centroid point, that is, the conjunction point of three bisectors of angles $\mathrm{AoD}, \mathrm{oAB}$, and $\mathrm{ABo}$, as shown in Fig. 1. Likewise, the vortex center in the region of oDCB can be assumed to be the meeting point of bisectors of DoC and $\mathrm{oCB}$, and this center point should be limited in the region oGD. Thus, the vortex center for a channel with an arbitrary aspect ratio can be determined. Table 1 shows the comparison between measured and predicted centers in oAD and DoCB. From Table 1, one may conclude that the predicted values agree well with the measured central points, around which a multitude of fluid particles move.

To estimate the near-bed secondary velocity, eq. [32] is used. In the region of DGCB, Tracy's (1965) experimental data shows $S_{1}=S$ (Fig. 2), thus eq. [32] becomes

[33] $\quad w=\frac{v_{\mathrm{t}}}{u} \frac{\partial u}{\partial z}$

It can be inferred from eq. [33] that the quantity of $w$ reduces gradually from the corner to the central line as $\partial u / \partial z$ approaches to zero, at point $\mathrm{C}$ the velocity $w$ must be zero for $\mathrm{BC}$ is the symmetric line and $\partial u / \partial z=0$. In the region GDBC, the largest $w$ exists at the point $\mathrm{G}$, and then decreases to zero.

Equation [33] shows that no secondary currents appear, if the wall-tangent velocity gradient $\partial u / \partial z=0$. In circular pipe flows, tangential velocity variation is zero, therefore, no secondary currents exist in full circular pipe flow. However, for partially full circular channel, the boundary shear stress or local shear velocity is a variable along the wall (Knight and Sterling 2000). This leads to the nonzero near-wall velocity gradient in the direction tangent to wall, and eq. [33] pre- 
Table 1. Comparison of measured and predicted locations of central point " $\mathrm{o}_{1}$ " and " $\mathrm{O}_{2}$ ".

\begin{tabular}{|c|c|c|c|c|}
\hline Author & & Measured $(y / h, z / h)$ & Predicted $(y / h, z / h)$ & Remarks \\
\hline \multirow[t]{2}{*}{ Nezu et al. (1985) } & Central point " $\mathrm{o}_{1}$ " & $(0.6,0.3)$ & $(0.68,0.32)$ & Water channel, $b / h=5$ \\
\hline & Central point " $\mathrm{O}_{2}$ " & $(0.4,1.2)$ & $(0.43,1.21)$ & \\
\hline \multirow[t]{2}{*}{ Tracy (1965) } & Central point " $\mathrm{O}_{1}$ " & $(0.6,0.3)$ & $(0.68,0.32)$ & Smooth wind tunnel, $b / h=6.4$ \\
\hline & Central point " $\mathrm{O}_{2}$ " & $(0.4,1.1)$ & $(0.43,1.22)$ & \\
\hline \multirow{2}{*}{$\begin{array}{l}\text { Nezu and Nakagawa } \\
(1993 ; \text { p. 102) }\end{array}$} & Central point " $\mathrm{O}_{1}$ " & $(0.65,0.3)$ & $(0.71,0.29)$ & Smooth water channel, $b / h=1$ \\
\hline & Central point " $\mathrm{O}_{2}$ " & $(0.3,0.65)$ & $(0.29,0.71)$ & \\
\hline \multirow[t]{2}{*}{ Sata et al. (1994) } & Central point " $\mathrm{o}_{1}$ " & $(0.61,0.18)$ & $(0.71,0.29)$ & Smooth wind tunnel, $b / h=1$ \\
\hline & Central point " $\mathrm{O}_{2}$ " & $(0.18,0.83)$ & $(0.29,0.71)$ & \\
\hline \multirow{2}{*}{$\begin{array}{l}\text { Pettersson Reif and An- } \\
\text { dersson (2002) }\end{array}$} & Central point " $\mathrm{o}_{1}$ " & $(0.6,0.246)$ & $(0.71,0.29)$ & Numerical model, $b / h=1$ \\
\hline & Central point " $\mathrm{O}_{2}$ " & $(0.25,0.625)$ & $(0.29,0.71)$ & \\
\hline \multirow{2}{*}{$\begin{array}{l}\text { Nezu and Nakagawa } \\
(1993 ; \text { p. 153) }\end{array}$} & Central point " $\mathrm{o}_{1}$ " & $(0.65,0.25)$ & $(0.60,0.22)$ & Smooth water channel, $b / h=0.5$ \\
\hline & Central point " $\mathrm{O}_{2}$ " & $(0.15,0.3)$ & $(0.159,0.34)$ & \\
\hline
\end{tabular}

dicts that in such a case, secondary currents must appear, and this inference is consistent with Replogle and Chow's (Replogle and Chow 1966) measurement.

The direction of near-bed secondary velocity can be determined from eq. [33], which states that secondary currents in the region $\mathrm{oDBC}$ will be rightward (positive $w$ ) for $\partial u / \partial z>$ 0 . It is obvious that the flow is stagnant at point $o$, and the near-bed velocity $u$ at the point $\mathrm{G}$ is greater than that at point $\mathrm{o}$; thus, in the near-bed layer, there exists a rightward flow along the bed due to $\partial u / \partial z>0$, or a counterclockwise secondary current can be observed in the region oDBC. It can be interpreted from eq. [33] that the lateral variation of velocity $u$ is the source of secondary current, it leads directly to the nonzero wall-tangent velocity $w$, and the direction of $w$ always points to the location with higher near-bed velocity $u$; subsequently, the near-bed $w$ drives a circulation in the region enclosed by lines with zero $\tau$. Therefore, in the region $\mathrm{OAD}$ exists a clockwise vortex because the velocity $u$ near point " $A$ " is higher than the velocity near point $\mathrm{o}$, and the near-wall secondary velocity always moves toward the place with higher velocity $u$, i.e., from o to A.

By placing an artificial ridge on a bed of wide channel, Nezu and Nakagawa (1993) observed the secondary currents induced by the "ridge", and Fig. 9 shows their measured secondary currents. It is understandable that the near-bed velocity $u$ above the ridge is higher than that on the trough in Fig. 9 because the flow rate per unit width could be assumed to be constant, then the profile with smaller water depth corresponds to higher velocity. Equation [33] predicts that in Fig. 9, the nonzero $w$ would move toward the ridge from both sides, and two symmetrical secondary currents would be induced and the division line would be a vertical line through atop of the ridge, if the sidewall effect is negligible. As the ridge located on the edge of secondary cell induced by the sidewall, the sum of two vectors fields induced by the sidewall and the ridge leads to the asymmetric secondary currents, as shown in Fig. 9. Hence, eq. [33] can well explain the measurement shown in Fig. 9.

The near-bed velocity $w$ at point $\mathrm{G}$ is discussed, in the viscous sublayer, the velocity can be expressed as follows:

$$
\frac{u}{u_{*}}=\frac{u_{*} y}{v}
$$

Hence, for the velocity $u_{\delta}=u *^{2} \delta / \nu$, and $u *^{2}=\tau_{\mathrm{b}(\mathrm{z})} / \rho$, one gets $\partial \tau_{\mathrm{b}(\mathrm{kh})} / \partial z=\rho g S / k$ because of $-\left(\overline{u^{\prime} v^{\prime}}\right)_{\mathrm{o}}=0$ at point $\mathrm{D}$ and $S_{1}=S$ by using eq. [22]. Therefore, by using eq. [33], one can determine the wall-tangent velocity $w$ at the point $\mathrm{G}$ as

$$
\text { [35] } \quad \frac{w_{\mathrm{G}}}{u_{*}}=\frac{v_{\mathrm{t}}}{k u_{*} h}
$$

Webel and Schatzmann (1984) measured the lateral eddy viscosity in a smooth rectangular channel, and they found that $v_{\mathrm{t}} /(u * h)$ is constant and equal to 0.177 , thus,

[36] $\quad \frac{w_{\mathrm{G}}}{u_{*}}=\frac{0.177}{k}$

In laboratory, the ratio of bulk velocity to the shear velocity $u_{*}$ is generally around 15 to 20 (Nezu and Nakagawa 1993). It can be estimated from eq. [36] that secondary velocity amounts to one percent of the bulk velocity, thus indicating that eq. [36] is consistent with experimental results and eq. [33] is reasonable.

\section{Conclusions}

The underlying mechanisms that initiate secondary flow in developing turbulent flow along a corner has been investigated and presented. The main results obtained from the investigations can be summarized as follows:

The total shear stress $\tau$ on an interface can be defined as the time-averaged product of instantaneous streamwise velocity $U$ and the velocity $V_{n}$ normal to the interface, or the correlation of velocities in different directions is also a kind of shear stress. The lines with zero total shear stress delineate the region of secondary flow, no mean flow penetrates the lines with zero $\tau$, and the secondary currents are separated by the lines with zero $\tau$.

The flow region is divisible as presumed by Prandtl (1926) and Keulegan (1938), however, the division line that separates the neighboring secondary currents is not always identical to the bisector of the corner. For a very wide channel, the width of near sidewall secondary cell is up to $1.26 h$, which is different from the commonly accepted assumption, i.e., bisector division or $1.0 \mathrm{~h}$. 
The boundary always receives and dissipates the turbulent energy from the adjacent flow volume or the turbulent energy in any unit fluid volume is transported toward the nearest boundary to dissipate into the thermal energy, and along the same path of energy transport, the Reynolds shear stress must be linear (see eq. [23], i.e., along the normal direction of the boundary to the division line.

It is well known that the near-bed flow layer is the primary source of turbulence as small eddies are generated by the wall-normal gradient of streamwise velocity, and the small eddies depart from the boundary and rises into the main flow. This study reveals that this layer is also the source of large eddy or secondary flow if there exists a wall-tangent gradient of streamwise velocity $u$. In other words, the transverse variation of velocity $u$ is the sufficient condition for the existence of secondary currents, the direction of near-bed secondary flow $w$ always points toward the location with higher near-bed $u$, and the near-bed flow $w$ drives a circulation within the region bounded by zero $\tau$.

The relation between the lateral variation of streamwise velocity and the near-bed transverse velocity $w$ is obtained, the velocity $w$ is proportional to the shear velocity. The proportionality factor varies from 0.14 to 0.17 , depending on the aspect ratio.

The most important features of secondary currents, i.e., the vortex center, near-bed secondary velocity, and the size of vortex can be well modeled, which provides a simple tool for engineers to assess their practical problems.

\section{References}

Aly, A.M.M., Trupp, A.C., and Gerrard, A.D. 1978. Measurements and prediction of fully developed turbulent flow in an equilateral triangular duct. Journal of Fluid Mechanics, 85(1): 57-83. doi:10.1017/S0022112078000531.

Bottaro, A., Galletti, B., and Soueid, H. 2006. Formation of secondary vortices in turbulent square-duct flow. AIAA Journal, 44(4): 803-811. doi:10.2514/1.17327.

Boussinesq, J. 1877. Essai sur la théorie des eaux courantes. Mémoires Présentés par Divers Savants à l'Académie des Sciences, 23: $1-680$.

Brundrett, E., and Baines, W.D. 1964. The production and diffusion of vorticity in duct flow. Journal of Fluid Mechanics, 19(03): 375-394. doi:10.1017/S0022112064000799.

Chien, N., and Wan, Z. 1999. Mechanics of sediment transport. ASCE, Reston, Va.

Einstein, H.A. 1942. Formulas for the transportation of bed load. Transactions ASCE, 107: 133-169.

Einstein, H.A., and Li, H. 1958. Secondary currents in straight channels. Transactions - American Geophysical Union, 39(6): 1085-1088.

Faisst, H., and Eckhardt, B. 2003. Traveling waves in pipe flows. Physical Review Letters, 91(22): 224502. doi:10.1103/ PhysRevLett.91.224502.

Galletti, B., and Bottaro, A. 2004. Large-scale secondary structures in duct flow. Journal of Fluid Mechanics, 512: 85-94. doi:10. 1017/S0022112004009966.

Gavrilakis, S. 1992. Numerical simulation of low-Reynolds number turbulent flow through a straight square duct. Journal of Fluid Mechanics, 244(-1): 101-129. doi:10.1017/S0022112092002982.

Gerard, R. 1978. Secondary flow in noncircular conduits. Journal of the Hydraulics Division, 104: 755-773.

Gessner, F.B. 1973. The origin of secondary flow in turbulent flow along a corner. Journal of Fluid Mechanics, 58(01): 1-25. doi:10.1017/S0022112073002090.

Gessner, F.B., and Emery, A.F. 1981. The numerical prediction of developing turbulent flow in rectangular ducts. Journal of Fluids Engineering, ASME, 103: 445-455.

Hangen, R.L., and Dhanak, A.M. 1966. Momentum transfer in turbulent separated flow past a rectangular cavity. Journal of Applied Mechanics, ASME, 33(3): 641-646.

Hinze, J.O. 1973. Experimental investigation of secondary currents in the turbulent flow through a straight conduit. Applied Scientific Research, 28: 453-465.

Imamoto, H., and Ishigaki, T. 1988. Measurement of secondary flow in an open channel. In Proceedings of the 6th IAHR-APD Congress, Kyoto, Japan. pp. 513-520.

Keulegan, C.H. 1938. Laws of turbulent flow in open channels. Journal of Research of the National Bureau of Standards, 21: 707-740.

Knight, D.W., and Sterling, M.S. 2000. Boundary shear in circular pipes running in partially full. Journal of Hydraulic Engineering, 126(4): 263-275. doi:10.1061/(ASCE)0733-9429(2000) 126:4(263).

Knight, D.W., and Patel, H.S. 1985. Boundary shear in smooth rectangular ducts. Journal of Hydraulic Engineering, 111(1): 29-47. doi:10.1061/(ASCE)0733-9429(1985)111:1(29).

Knight, D.W., Demetriou, J.D., and Hamed, M.E. 1984. Boundary shear in smooth rectangular channels. Journal of Hydraulic Engineering, 110(4): 405-422. doi:10.1061/(ASCE)07339429(1984)110:4(405).

Lim, S.Y., and Yang, S.Q. 2005. Simplified model of tractiveforce distribution in closed conduits. Journal of Hydraulic Engineering, 131(4): 322-329. doi:10.1061/(ASCE)0733-9429(2005) 131:4(322)

Liu, Q.Q. 2006. Laboratory study on sediment diffusion and deposition into blind channels. Advances in Water Resources, 29(12): 1804-1814. doi:10.1016/j.advwatres.2006.01.001.

Launder, B.E., and Ying, W.M. 1972. Secondary flows in ducts of square cross section. Journal of Fluid Mechanics, 54(02): 289295. doi:10.1017/S0022112072000680.

Madabhushi, S., and Vanka, S.P. 1991. Large eddy simulation of turbulence-driven secondary flow in a square duct. Physics of Fluids. A, Fluid Dynamics, 3(11): 2734-2745. doi:10.1063/1.858163.

Melling, A., and Whitelaw, J.H. 1976. Turbulent flow in a rectangular duct. Journal of Fluid Mechanics, 78(02): 289-345. doi:10. 1017/S0022112076002450.

Mompean G., Gavrilakis S., Machiels L., and Deville M.O. 1996. On predicting the turbulence-induced secondary flows using nonlinear k-epsilon models. Physics of Fluids, 8(7): 1856-1868.

Mompean, G. 1998. Numerical simulation of a turbulent flow near a right-angled corner using the Speziale non-linear model with RNG k-epsilon equations. Computers \& Fluids, 27(7): 847-859.

Nezu, I., and Nakagawa, H. 1984. Cellular secondary currents in straight conduit. Journal of Hydraulics Engineering, ASCE, 110(2): 173-193. doi:10.1061/(ASCE)0733-9429(1984)110:2(173).

Nezu, I., and Nakagawa, H. 1993. Turbulence in open-channel flows. Balkema, Rotterdam, The Netherlands.

Nezu, I., Nakagawa, H., and Tominaga, A. 1985. Secondary currents in a straight channel flow and the relation to its aspect ratio. In Turbulent Shear Flows 4. Springer-Verlag. pp. 246-260.

Nikuradse, J. 1926. Untersuchungen über die Geschwindigkeitsverteilung in turbulenten Strömungen. Ph.D. thesis, University of Göttingen, Germany.

Perkins, H.J. 1970. The formation of streamwise vorticity in turbulent flow. Journal of Fluid Mechanics, 44(04): 721-740. doi:10. 1017/S0022112070002112. 
Pettersson Reif, B.A., and Andersson, H.I. 2002. Prediction of turbulence-generated secondary mean flow in a square duct. Flow, Turbulence and Combustion, 68(1): 41-61. doi:10.1023/ A:1015611721026.

Prandtl, L. 1926. Über die ausgebildete Turbulenz. In Proceedings of the Verh. 2nd International Kong. Trans. N.A.C.A. Tech. Momo. No.435. Für Tech. Mech., Zürich. pp. 62-75.

Replogle, J.A., and Chow, V.T. 1966. Tractive-force distribution in open channels. Journal of the Hydraulics Division, 92(2): 169191.

Rhodes D.G., Knight D.W., 1994. Distribution of shear force on boundary of smooth rectangular duct. Journal of Hydraulic Engineering, ASCE, 120(7): 787-807

Sata, Y., Sato, K., Kasagi, N., and Takamura, N. 1994. Turbulent air flow measurement with three-dimensional particle tracking velocimetry. Transaction of JSME Series B, 60(571): 865-871.

Schlichting, H. 1979. Boundary layer theory. 7th ed. McGraw-Hill, New York. p. 614

Schmidt, J.C. 1990. Recirculating flow and sedimentation in the Colorado River in Grand Canyon, Arizona. The Journal of Geology, 98: 709-724.

Speziale, C.G. 1982. On turbulent secondary flows in pipes of noncircular cross section. International Journal of Engineering Science, 20(7): 863-872. doi:10.1016/0020-7225(82)90008-8.

Speziale, C.G. 1987. On non-linear k-1 and k- $\varepsilon$ models of turbulence. Journal of Fluid Mechanics, 91(6): 9-35.

Tracy, H.J. 1965. Turbulent flow in a three-dimensional channel. Journal of Hydraulic Engineering, 91(6): 9-35.

van Schijndel, S.A.H. 1998. Reducing the siltation of a river harbour. Journal of Hydraulic Research, 36(5): 803-814.

Webel, G., and Schatzmann, M. 1984. Transverse mixing in open channel flow. Journal of Hydraulic Engineering, 110(4): 423435. doi:10.1061/(ASCE)0733-9429(1984)110:4(423).

Yang, S.Q. 1993. The law of boundary shear in rectangular open channels. Journal of Sediment Research, Beijing, 3: 95-103. [In Chinese.]

Yang, S.Q., and Lim, S.Y. 1997. Mechanism of energy transportation and turbulent flow in a $3 \mathrm{D}$ channel. Journal of Hydraulic Engineering, 123(8): 684-692. doi:10.1061/(ASCE)07339429(1997)123:8(684).

Yang, S.Q., and McCorquodale, A.J. 2004. Determination of boundary shear stress and Reynolds shear stress in smooth rectangular channel flows. Journal of Hydraulic Engineering, 130(5): 458-462. doi:10.1061/(ASCE)0733-9429(2004) 130:5(458).

Yang, S.Q., Yu, J.X., and Wang, Y.Z. 2004. Estimation of diffu- sion coefficients, lateral shear stress, and velocity in open channels with complex geometry. Water Resources Research, 40(5): W05202. doi:10.1029/2003WR002818.

Yang, S.Q., Tan, S.Q., and Lim, S.Y. 2005. Flow resistance and bed form geometry in a wide alluvial channel. Water Resources Research, 41(9): W09419. doi:10.1029/2005WR004211.

Yang S.Q., and Lim, S.Y. 2006. Discussion on shear stress in smooth rectangular open-channel flows. Journal of Hydraulic Engineering, ASCE, 132(6): 629-631.

\section{List of symbols}

$A_{s}$ area

$b$ half width of channel

$\boldsymbol{e}$ unit vector

$h$ half height of channel

$k$ slope of division line

$\boldsymbol{n}$ normal direction

$s$ curve

$S$ energy slop

$U$ instantaneous velocity in $x$-direction

$u$ time averaged velocity in $x$-direction

$u *$ shear velocity

$u^{\prime}, v^{\prime}$, and $w^{\prime}$ turbulent velocity fluctuations

$u_{\delta}$ velocity at viscous sublayer

$v$ time-average velocities in $z$ directions

$\boldsymbol{V}$ instantaneous velocity of secondary flow

$v_{\mathrm{o}}, w_{\mathrm{O}}$ components of secondary current along the division line

$V_{\mathrm{t}}$ component of $\boldsymbol{V}$ on the tangent direction

$\bar{u}_{* \mathrm{~b}} \quad \sqrt{\bar{\tau}_{\mathrm{b}}} / \rho$

$\bar{u}_{* \mathrm{w}} \sqrt{\bar{\tau}_{\mathrm{w}}} / \rho$

$w$ time-average velocities in $z$ directions

$y_{\mathrm{O}}, z_{\mathrm{o}}$ normal distance from boundary to division line

$v_{\mathrm{t}}$ transverse eddy viscosity

$\rho$ fluid density

$\kappa$ Karman constant

$\rho g S_{1}, \rho g S_{2}$ gradients of the measured Reynolds shear stress in $y$ and $z$ directions

$\tau$ shear stress

$-\overline{u^{\prime} v^{\prime}},-\overline{u^{\prime} w^{\prime}}$ Reynolds shear stress

$\tau_{x y} \quad \mu \partial u / \partial y-\rho \overline{u^{\prime} v^{\prime}}$

$\tau_{x z} \mu \partial u / \partial z-\rho \overline{u^{\prime} w^{\prime}}$

$\mu$ dynamic viscosity

$v$ kinematical viscosity 